\title{
HPTLC AND GC-MS Analysis of Methanolic Extracts of Samanea Saman (Albizia Saman) (Jacquin) F. Mueller
}

\author{
S. D. Shanmugakumar ${ }^{1}$, G.Satheesh kumar $^{2} \&$ K.Padmalatha ${ }^{1}$ \\ ${ }^{I}$ Vijaya Institute of Pharmaceutical sciences for women, Enikepadu, Vijayawada Rural \\ Vijayawada-521108. Andhra Pradesh \\ ${ }^{2}$ Jyothishmathi College of Pharmacy, Turkapally (V), Shamirpet (M), R.R.District -500078. Telangana
}

\begin{abstract}
Samanea saman (Albizia saman) is a wide-canopied tree with a large symmetrical crown. It usually reaches a height of $25 \mathrm{~m}(82 \mathrm{ft})$ and a diameter of $40 \mathrm{~m}$. The leaves fold in rainy weather and in the evening, hence the name "rain tree" and "five o'clock tree" (Pukul Lima) in Malay. In the present investigation, the bioactive methanolic fraction was subjected to HPTLC and GC-MS analysis. GC-MS analysis revealed the presence of six compounds from the methanolic fraction of the Samanea saman (1) 9,Octadecenoic acid (Z) methyl ester, (2) Dodecanoic acid, 10-methyl, methyl ester, (3) 13- Hexyloxyacylotridec-10-en-2-one, (4) 15Octadecenoic acid, methyl ester (5) Diethyl -1- (8 -amino -1- naphthyl) -1,2,3 triazole -4,5-dicarboxylate were identified from the methanolic extract from the leaves of Samanea saman. The presence of these compounds was confirmed by NIST library values. HPTLC analysis concludes the presence of four major phytoconstituents which was eluted at $R_{f} 0.67 ; 0.79 ; 0.85 \& 0.90$ respectively.
\end{abstract}

Key words: Samanea saman (Albizia saman); HPTLC analysis; GC-MS analysis.

\section{Introduction}

Medicinal plants play a vital role in rescuing the various ailments caused in the human arena. Natural product could be a potential drug for human and live stock and their analogues can acts as an intermediate for the synthesis of useful drugs. Folklore claims stated that there were many unsung biological potentials were present in the plant Samanea saman (Albizia saman) which belong to the family fabaceae, sub family Mimosoideae. Samanea saman (Albizia saman) is an ever green umbrella-shaped canopy, which grows up to the height $15-25 \mathrm{~m}$ and it is a native to South America, Columbia, Mexico, Fiji and also cultivated in the plains of India. The synonym is a rain tree. The parts of the plant possess various medicinal properties like antioxidant, antiulcerogenic, antimicrobial, analgesic, antitubercular and cytotoxic activities [1-3]. The present work has been undertaken to evaluate the phytoconstituents present in the leaf extracts using Gas chromatography and mass spectrometer analysis.

\section{Plant Materials}

\section{Material and Methods}

The leaves of Samanea saman (Albizia saman) were collected from Turkapally -Village, Shamirpet Mandal, Ranga reddy district. Care was taken to select the healthy plants and for normal organs (i.e. leaves, flowers, fruits and seeds).The required parts were cut and removed from the plant after proper identification and authentication has done by the National Institute of Herbal research, Chennai, Tamil Nadu (PARC/2014/858).

\section{Extract Preparation and column chromatographic analysis}

The air -dried leaves of Samanea saman was thoroughly washed with water and then shade dried. The shade dried leaves were being pulverized in to coarse powder. The coarse powder of the dried leaves were subjected to cold maceration based on increasing polarity (Hexane, Chloroform and Methanol). The extracts were concentrated using a rota vap in vасио. The corresponding percentage of extracts were $0.5 \%, 1.2 \%$ \& $1.6 \%$. The extracts were subjected for biological screening to explore the unsung biological potentials. Bioactive methanolic extract $(1.6 \%)$ of Samanea saman was fractionated over a silica gel (100-200 mesh) column by eluting with solvents of increasing polarity. The fractions obtained were subjected to TLC analysis. The similar fractions were been combined as monitored by the TLC. The obtained fractions were subjected to phytochemical analysis to explore their phytoconstituents. The fraction (ethyl acetate : methanol 80:20) were concentrated using a rota vap in vacuo. The obtained brown oily liquid were subjected to HPTLC analysis and GC- MS analysis to explore the bioactive phyto constituents.

HPTLC analysis

HPTLC is a modern adaptation of TLC with improved versatility, separation efficiency and detection limits. It is a reliable method for quantization of nanogram level in a complex formulation. Camag TLC scanner 3" Scanner 
$3-070408$ “ S/N 070408 (1.14.21) was used for detection and CAMAG Linomat 5 sample applicator was used for the application of the tracks [4].

Sample preparation: The bioactive methanolic fraction $(20 \mu \mathrm{L})$ of Samanea saman were loaded in the CAMAG Linomat 5 sample applicator. The sample were coated in the aluminum sheets pre-coated with silica gel $\mathrm{G}_{254}$ (Merck), $0.2 \mathrm{~mm}$ layer thickness. The slit dimensions $5 \mathrm{mmx} 0.45 \mathrm{~mm}$ and scanning speed of $20 \mathrm{~mm} / \mathrm{sec}$ was employed.

Mobile phase: The chromatogram was developed using N-hexane: Ethyl acetate $(60: 40 \mathrm{v} / \mathrm{v})$ as a mobile phase. Detector: Densitometric scanning was performed on CAMAG TLC scanner at $420 \mathrm{~nm}$ operated by WINCATS software version 1.14.21.

\section{GC-MS analysis}

GC-MS analysis [5] was carried out on a perkin elmer clarus 680 GC-MS instrument employing the following condition : column elite -5MS $(30.0 \mathrm{~m}, 0.25 \mathrm{~mm}$ ID, $250 \mu \mathrm{m}$, operating electronic impact mode; helium was used as a carrier gas at a constant flow and split ratio is $10: 1$; injector temperature is $250^{\circ} \mathrm{C}$; flow rate is $1 \mathrm{~mL} / \mathrm{min}$; oven temperature is $60^{\circ} \mathrm{C}$ for $3 \mathrm{~min}$, ramp $20^{\circ} \mathrm{C} / \mathrm{min}$ to $500^{\circ} \mathrm{C}$ hold for $6 \mathrm{~min}$. The total run time is $35.00 \mathrm{~min}$. The molecular weight and structure of the compounds were ascertained by interpretation using the data base of National Institute of Science and Technology (NIST).

\section{HPTLC analysis}

\section{Results and Discussion}

Conventional extraction of methanolic Samanea saman adopted in this work reveals the presence of 4 bioactive components with the corresponding $\mathrm{Rf}$ values $0.67,0.79,0.85 \& 0.90$ respectively. The $\%$ of area of the corresponding peaks were been tabulated in the Table 1.[Figure 1].

\section{GC-MS analysis}

The GC-MS chromatogram of methanolic Samanea saman extract gave 6 peaks which are shown in Figure 2. The active principle with their retention time, molecular weight and structure are shown in Table 2. Six compounds were detected in the methanolic extract of Samanea saman. The GC spectral study revealed the presence of six compounds include : (1) 9-octadecenoic acid- RT 17.12, (2) Dodecanoic acid,10 - methyl methyl ester- RT 18.5, (3) 13-Hexyloxacyclotridec-10-en-2-one - RT 18.83, (4) 15- octa decenoic acid methyl ester - RT 20.52, (5) Diethyl 1-(8-amino -1-naphthyl) -1,2,3 triazole -4,5 -dicarboxylate - RT 22, (6) N.N [1,4 -butanediyl bis [ ethylimino] -3,1 propane diyl] bis[ N-ethyl acetamide]- RT 24.1 respectively.

\section{Conclusion}

The conventional method of extraction (Cold maceration) is a simple, inexpensive and also avoids the decomposition of thermo labile substances which are bioactive in nature. In the present study, four major components were been identified from the methanolic extract of Samanea saman by HPTLC analysis. GC-MS analysis identifies the presence of six compounds in which N.N - [1,4 -butanediyl bis [ethylimino] -3,1 propane dily] bis [ N- ethyl acetamide] was first reported in the leaf extract of Samanea saman. The present analytical study would explore the unsung bio molecules and provide a new arena for the discovery of modern drug molecules.

\section{Acknowledgement}

The authors are thankful to the sophisticated analytical instrument facility (SAIF), Indian Institute of Technology, Chennai for providing necessary facilities for the GC-MS analysis. Further, authors were very grateful to NISHKA scientific \& reference laboratories, Hyderabad for helping in the HPTLC finger print analysis.

\section{References}

[1]. Faisol Ferdous, Khalid Hossain, Md. Rahman, S and Rashid, A., Chemical and Biological investigations of Samanea saman (Jacq.) Merr, Dhaka University. J.Pharm.Sci , 9(2),2010,69-73.

[2]. Ravikumar, A. Rijwana shaik and Yeshwanth, D., Phytochemical evaluation of Delonix Regia and Samanea saman, International Journal of research in Pharmacy and chemistry, 3(4),2013, 5-10.

[3]. Iqbal Azhar, Moht asheemul hasan, M and Ali, M.S., Some biological evaluations on Samanea saman, Pakistan Journal of Pharmacology, 26 (1),2009, 47-53.

[4]. Arul priya, P. Lalitha, P and Hemalatha, S., HPTLC finger print of ethyl acetate extract of Samanea saman (Jacq.) Merr, Asian Journal of Research in Chemistry, 4(2), 2011, 266-269.

[5]. Preethi, S and Mary sarai, A., GC-MS analysis of microwave assisted ethanolic extract of Pithecellobium dulce, Malaya Journal of Biosciences, 1(4), 2014, 242-247. 
HPTLC AND GC-MS Analysis Of Methanolic Extracts Of Samanea Saman (Albizia Saman) (Jacquin)

Table 1 : HPTLC data of Methanolic extract of Samanea saman

\begin{tabular}{|c|l|c|c|c|c|c|l|l|r|}
\hline Peak & Peak & Peak & Max & Max & Max \% & End & End & Area & Area \% \\
No & strat & position & position & height & & position & height & & \\
\hline 1 & $0.67 \mathrm{Rf}$ & $0.2 \mathrm{AU}$ & $0.72 \mathrm{Rf}$ & $155.2 \mathrm{AU}$ & $19.56 \%$ & $0.78 \mathrm{Rf}$ & $0.6 \mathrm{AU}$ & $4843.3 \mathrm{AU}$ & $20.36 \%$ \\
\hline 2 & $0.79 \mathrm{Rf}$ & $0.3 \mathrm{AU}$ & $0.83 \mathrm{Rf}$ & $182.1 \mathrm{AU}$ & $22.95 \%$ & $0.85 \mathrm{Rf}$ & $76.4 \mathrm{AU}$ & $4836.1 \mathrm{AU}$ & $20.33 \%$ \\
\hline 3 & $0.85 \mathrm{Rf}$ & $76.5 \mathrm{AU}$ & $0.88 \mathrm{Rf}$ & $243.9 \mathrm{AU}$ & $30.74 \%$ & $0.90 \mathrm{Rf}$ & $164.0 \mathrm{AU}$ & $7693.9 \mathrm{AU}$ & $32.35 \%$ \\
\hline 4 & $0.90 \mathrm{Rf}$ & 165.2 & $0.92 \mathrm{Rf}$ & $200.2 \mathrm{AU}$ & $25.23 \%$ & $0.97 \mathrm{Rf}$ & $2.1 \mathrm{AU}$ & $6253.9 \mathrm{AU}$ & $26.30 \%$ \\
& & $\mathrm{AU}$ & & & & & & & \\
\hline
\end{tabular}

FIGURE 1 HPTLCanalysis of Methanolic extract of Samanea saman
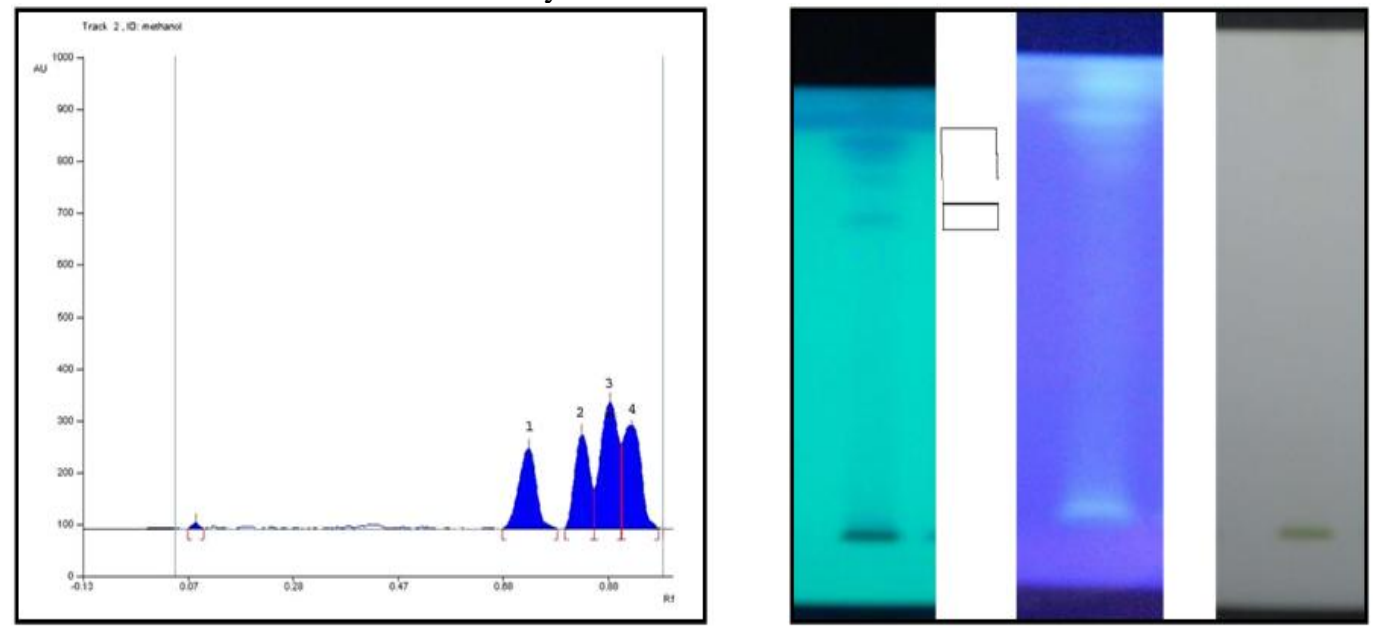

FIGURE 2 GC-MS Analysis of Methanolic extract of Samanea saman

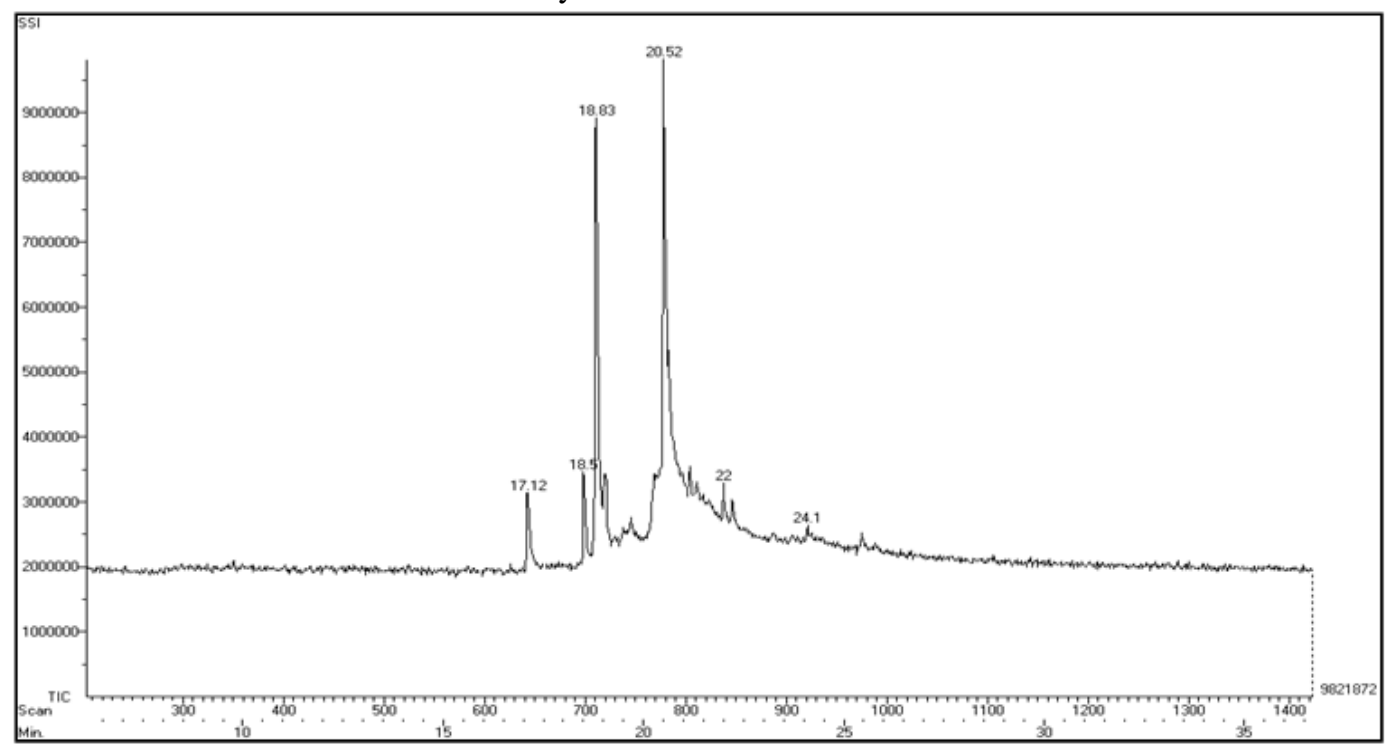



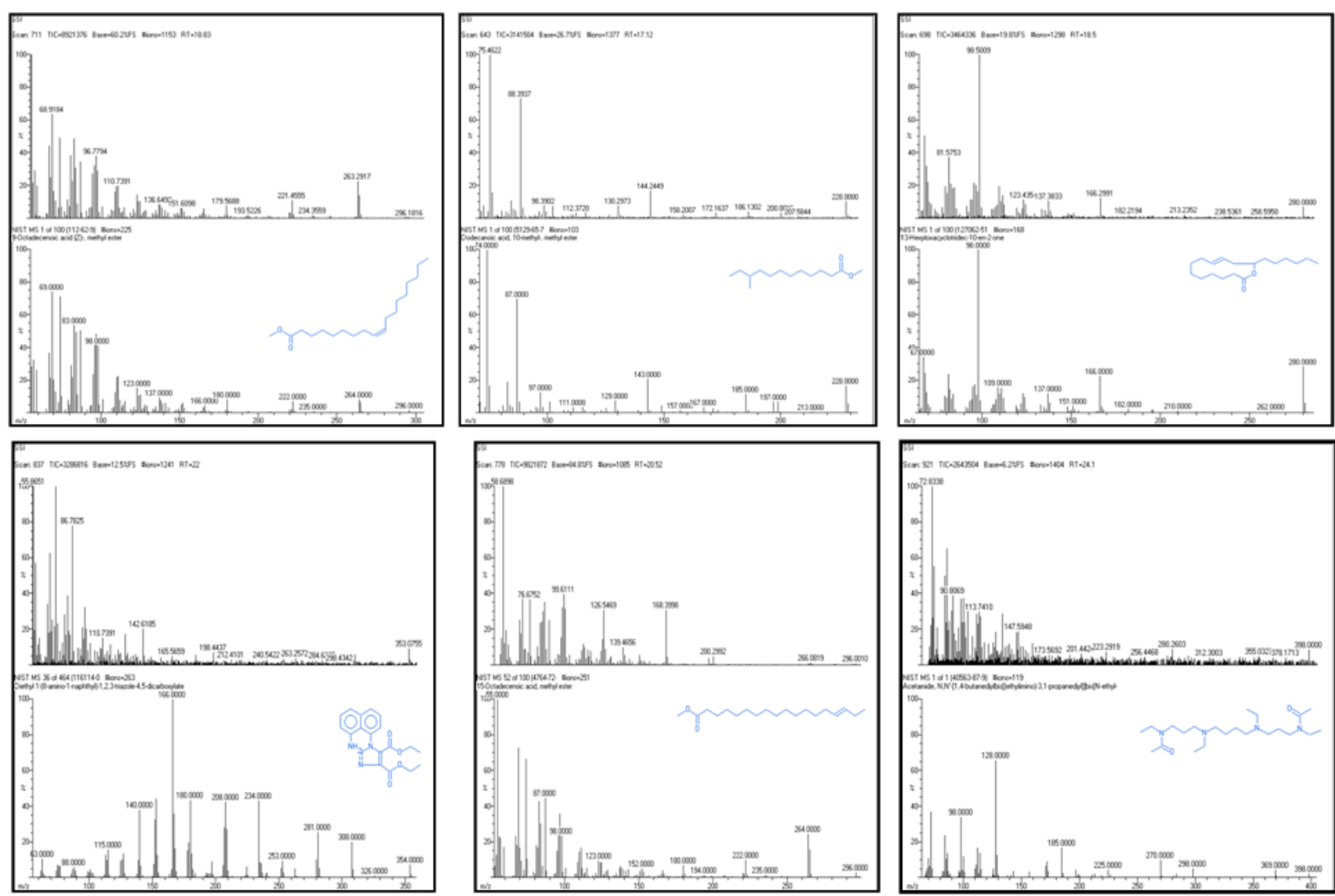